\title{
Die Anforderungen nehmen weiter zu
}

$\mathrm{D}$ as Jahr 2005 wird zeigen, dass die zunehmend offenbar werdenden Probleme im Gesundheitssystem uns allen weitere administrative und fachliche Aufgaben auferlegen werden sowohl in der Praxis als auch im Krankenhaus. Es ist bemerkenswert: Auf Seiten der staatlichen Institutionen glaubt man anscheinend, dass die Vermehrung von Papier gleich einer Verbesserung der Qualität der Patientenversorgung sei.

Jeder von uns, in Praxis, Krankenhaus oder Universitätsklinik, hat ohne Frage sowohl die Schwächen der gegenwärtigen Systeme als auch Lösungsansätze im Visier. Hierzu gehört ein Stufenkonzept der Versorgung allergischer Krankheiten durch Hausarzt, Facharzt, Krankenhaus. Die Vorstände der nationalen Fachgesellschaften DGAI und ÄDA haben hierzu vor wenigen Jahren ein klares Dreistufenkonzept mit dem Institut für Qualitätssicherung von Bundesärztekammer und Kassenärztlicher Bundesvereinigung erarbeitet. Bedenken seitens der Juristen des Instituts für Qualitätssicherung haben - vor allem im Hinblick auf die hausärztlichen Interessen -

„Auf Seiten der staatlichen Institutionen glaubt man anscheinend, dass die Vermehrung von Papier gleich einer Ver-

besserung der Qualität der Patientenversorgung sei.“ terhin erst einmal die alljährliche Gelegenheit, durch Fortbildung auf nationalen und internationalen Tagungen ihre Standpunkte zu vertiefen (siehe Termine auf S. 78).

Die Deutsche Gesellschaft für Allergologie und klinische Immunologie lädt ein zum 17. Mainzer Allergie-Workshop vom 11.-12. März 2005. Seit 17 Jahren trifft sich nunmehr die allergologisch interessierte junge wissenschaftliche Szene im Hörsaal der Mainzer Universitätshautklinik, um die Fortschritte der nationalen Forschung zu diskutieren. Der Fortschritt der Kompetenz ist beeindruckend. Aus dem Programm wird vor allem deutlich, dass die Forschungsfortschritte gebunden sind an optimale lokale Forschungsstrukturen. Hier ragen 2005 die Forschungsaktivitäten in München (TU und GSF München), das BGFA Bochum, das For-

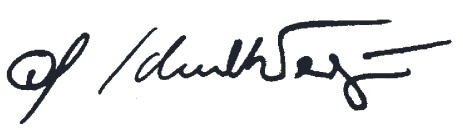

Prof. Dr. Gerhard Schultze-Werninghaus schungszentrum Borstel, das Institut für Immunologie Mainz, das Fraunhofer-Institut Hannover und die Klinik für Dermatologie und Venerologie Hannover heraus, andere Institutionen sind jedoch in beachtlicher Anzahl beteiligt.

Über den Mainzer Workshop hinaus wird 2005 ein besonderes Highlight die nationale Allergologie bestimmen: Dies ist der World Allergy Congress 2005 in München vom 26. Juni bis 1. Juli 2005. Der Kongress ist eine gemeinsame Veranstaltung der World Allergy Organization (WAO) und der European Academy of Allergology and Clinical Immunology (EAACI). Diese Veranstaltung wird von der DGAI und vom ÄDA nachhaltig unterstützt. Allen Mitgliedern wird eine Teilnahme sehr empfohlen. Anmeldungen können erfolgen unter www.congrex.com/wac2005.

„Wenn du ein Schiff bauen willst, so trommle nicht Leute zusammen, um Holz zu beschaffen, Werkzeuge vorzubereiten, Aufgaben zu vergeben und die Arbeit einzuteilen, sondern wecke in ihnen die Sehnsucht nach dem weiten, unendlichen Meer." Diesem Ausspruch von Saint-Exupéry sollten wir folgen, um an den uns möglichen Ansatzpunkten eine Änderung der derzeitigen Situation im Gesundheitswesen und eine Verbesserung der Versorgung bei gleichzeitiger Optimierung der Wirtschaftlichkeit herbeizuführen. Geschichte ist nicht ein zwangsläufiger Prozess, sondern das Ergebnis individueller Aktivitäten. Die Sehnsucht nach Verbesserungen unserer und Ihrer Arbeitsbedingungen möge uns alle 2005 bestimmen.

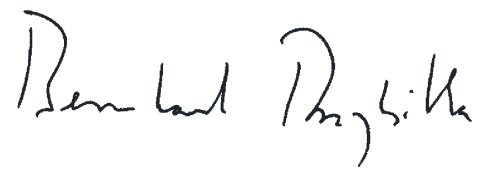

Prof. Dr. Bernhard Przybilla 